\title{
Evaluation of Functional Splits in terms of Optimal Number of Users Served in 5G Downlink
}

\author{
Pagadala Dinesh Kumar*, Kishore K R \\ Research Engineer \\ Centre for Development of Telematics \\ Bangalore, India \\ *pdk@cdot.in
}

\begin{abstract}
G is expected to provide reliable and high-speed broadband services for connecting, monitoring and controlling everything everywhere wirelessly. Millimeter Wave spectrum, massive Multiple Input Multiple Output (mMIMO), and Cloud Radio Access Network (C-RAN), are the key technologies which are expected to make $5 \mathrm{G}$ a reality. When massive MIMO is implemented in the context of C-RAN, the fronthaul capacity and the choice of functional split will limit the number of antennas that can be used. This effects the maximum achievable sum rate. In this paper, we estimate the maximum number of antennas that can be used at each functional split. Further, the optimal number of users that can be served at each functional split, with Zero-Forcing (ZF) \& Maximum Ratio Transmission (MRT) beamforming techniques is also investigated. It is observed that, split option 1 supports the maximum number of antennas, and serves the maximum number of users.
\end{abstract}

Keywords: Massive MIMO, C-RAN, Beamforming, 5G

\section{INTRODUCTION}

$5 \mathrm{G}$ relies on the key technologies like ultra-densification, millimeter Wave spectrum, mMIMO and C-RAN to meet its varied set of new use case requirements [1]. Implementation of new cellular technologies were always associated with an increase in capital and operational expenditures, but operators rarely witnessed any significant growth in average revenue per user. C-RAN solves this problem by avoiding underutilization of resources during low activity periods and thus stands as a cost-efficient solution for handling a large number of users and meeting their requirements [2]. In 2012, annual average power consumption of the Information and Communications Technologies industries was around 200 $\mathrm{GW}$, out of which telecoms infrastructure \& devices accounted for $25 \%$ [3]. The radio access network accounts for about $70 \%$ of the total power consumption of cellular networks. Due to the adverse effects of global warming, steps for minimizing the carbon footprint from cellular technologies has been recognized as an urgent issue across the world. In this regard, C-RAN stands as an eco-friendly technology by enabling efficient implementation of techniques like Carrier Aggregation and Coordinated MultiPoint (CoMP).

The BaseBand Unit (BBU) and Radio Unit (RU) were colocated and connected via lossy coaxial cables in the traditional base station architecture. As the technology pushed further, BBU and RU were separated, and BBU was placed at comparatively convenient location making maintenance and monitoring easier. Now, C-RAN proposes to group certain baseband functionalities at a Central Unit (CU) and place it in a cloud location, as shown in fig. 1. It is likely to have virtual BBU instances running on a physical machine. The major components of this architecture are [2]:
1. Central Unit: This entity comprises of baseband functionalities of many individual BBUs, which have high storage and computational capabilities. $\mathrm{CU}$ is responsible for processing and dynamically allocating resources to RUs based on the current network requirements.

2. Remote Radio Units: RU comprises of antennas, to get connected to the User Equipment (UEs). These RUs are placed at remote locations, far from the CU and hence, they are termed as Remote RUs (RRU)

3. Transport network: This is the link between CU and set of RRUs. It is called as fronthaul, and is required to provide high bandwidth to handle the requirements of multiple RRUs. Initially, optical fiber communication was considered to be ideal for C-RAN. Today there are many proposals for using ethernet as the fronthaul.

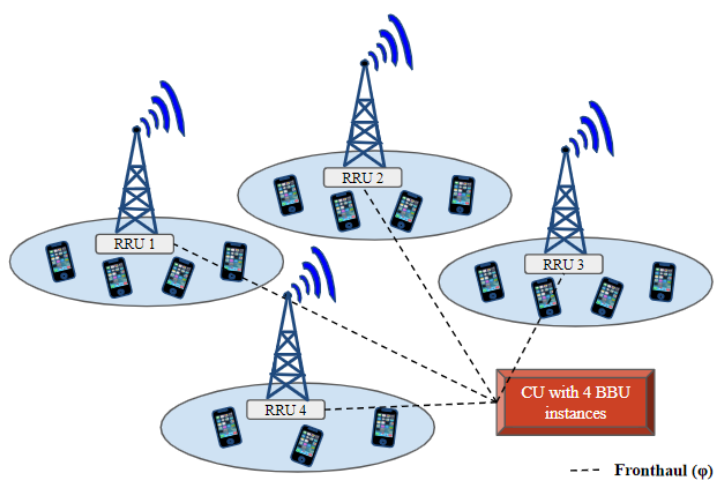

Figure 1: Components of the C-RAN architecture

C-RAN provides a better resource utilization, as the resources of individual BBUs are aggregated and shared in the pool. C-RAN can provide an efficient solution for the consequences of Tidal effect by pooling the BBUs with varied time geometric traffic patterns. By pooling the individual BBUs, power consumed by the air conditioners can be considerably reduced [2].

MIMO uses multiple antennas at both transmitter and receiver, to provides a reliable high-speed connection by, exploiting the wireless channel diversity. mMIMO further enhances the traditional MIMO, by using a very large number of antennas, to serve more UEs. By using a large number of antennas, there'll be an improvement in the link reliability, network throughput, capacity, and coverage [4]. Both CRAN and mMIMO independently provide a lot of advantages, but when mMIMO is implemented in the context of C-RAN, the fronthaul capacity and the choice of functional split will limit the number of antennas that can be used. This will in turn effect the maximum achievable sum rate. So, in 
this paper, we estimate the maximum number of antennas, and optimal number of users that can be served at each functional split, with both ZF and MRT beamforming.

This paper is organized as follows: the challenges of CRAN and possible solutions are explained in Section II, Section III describes the system model, Section IV describes the problem of wireless sum rate maximization, and the simulation results. Finally, section IV concludes the paper.

\section{Challenges OF C-RAN:}

Inefficiency of existing fronthaul network to support huge bandwidth is the major bottleneck for C-RAN. Commercial deployment of C-RAN will be successful only when there is a proper compensation for this inefficiency. Fronthaul in the traditional RANs was often a point to point connection, and the radio signals were transmitted using Common Public Radio Interface (CPRI). But, $5 \mathrm{G}$ need a flexible fronthaul to cater all the services efficiently. CPRI based fronthaul forwards the IQ data which requires large optical bandwidth (10 times more than original wireless data rate). With the advent of $5 \mathrm{G}$, there will be much higher data rates resulting in a very huge optical bandwidth requirement on fronthaul. This will not allow the C-RAN to be cost effective anymore.

Though there are various IQ compression techniques which can provide a compression ratio equal to 0.5 , they are not really sufficient to reduce the required bandwidth from several tens of Gbps to $10 \mathrm{Gbps}$ or less [5]. Other popular solution to this problem is to split and centralized the baseband functionalities at some point. 3GPP specifies eight functional split options as shown in fig. 2 .
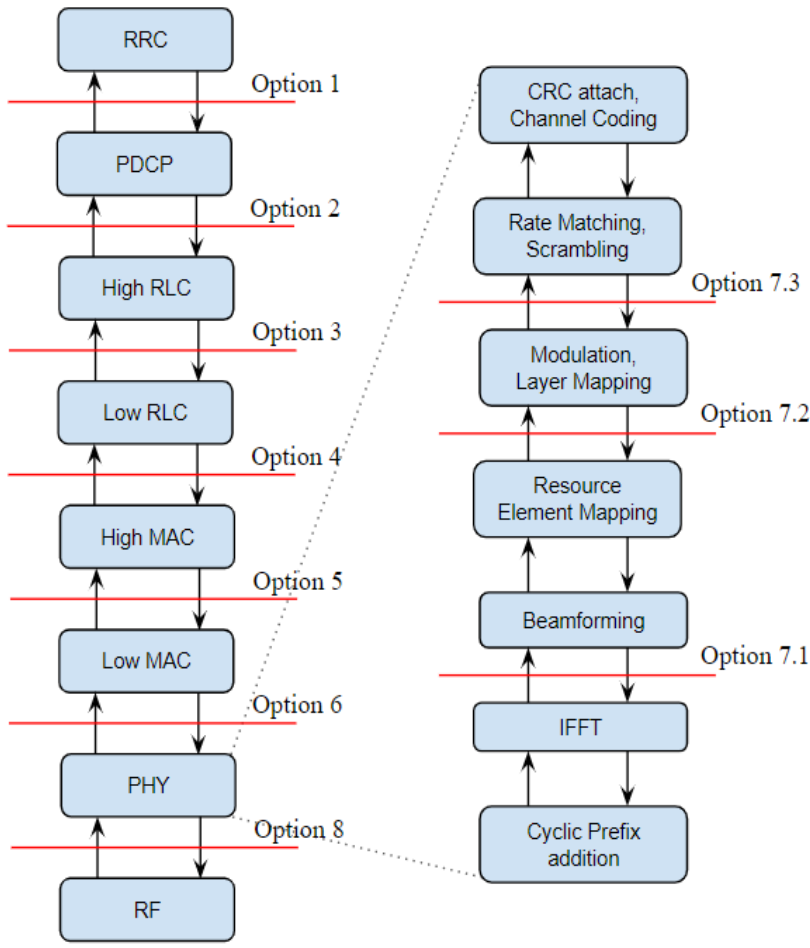

Figure 2: Functional Splits as specified by 3GPP

As we move towards split option 1 from split option 8 , bandwidth requirement reduces at the expense of number of functions that can be centralized. As a result, efficient implementation of Coordinated Multi Point and Carrier Aggregation becomes difficult. An exhaustive survey on the functional splits is provided in [6].

\section{SYSTEM MODEL}

The system model consists of a single cell with base station equipped with ' $M$ ' antennas to serve a maximum of ' $K$ ' single antenna users. The channel is considered to be a Rayleigh fading MIMO channel with the assumption of perfect channel state information. Here, $\boldsymbol{h}_{\boldsymbol{k}}$ denotes the channel vector between base station and user $k$, and $H$ is the channel matrix accommodating $\boldsymbol{h}_{\boldsymbol{k}}$ vectors of all the users. $\boldsymbol{w}_{\boldsymbol{k}}$ denotes beamforming vector of $k^{\text {th }}$ user, and $W$ denotes system beamforming matrix.

Beamforming refers to steering of transmitted signals to an intended angular direction by applying relative amplitude and phase shifts to each antenna element, such that output signals from antenna array add constructively for one particular angle and cancel out each other for other signals [7]. All the received signals are coherently combined at the receiver, using different scale factors, to maximize the SNR.

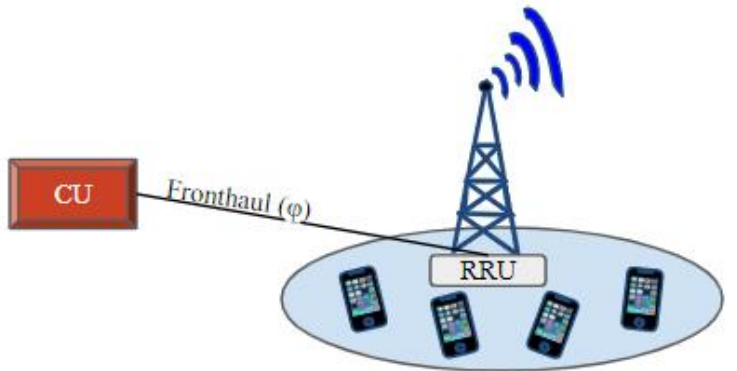

Figure 3: System Model

Consider $P$ is the total downlink transmit power, and if vector $\boldsymbol{x}$ is transmitted, then according to [8], the received vector $\mathbf{y}$ is given by:

$$
\boldsymbol{y}=\sqrt{P} H \boldsymbol{x}+n=\sqrt{P} H . W S+n
$$

Here, $S$ is the receiver signal matrix, and $\mathrm{n}$ is Additive White Gaussian Noise. Then, the signal received by the $k^{\text {th }}$ user is:

$$
\boldsymbol{y}_{\boldsymbol{k}}=\sqrt{P} \boldsymbol{h}_{\boldsymbol{k}} \boldsymbol{w}_{\boldsymbol{k}} \boldsymbol{s}_{\boldsymbol{k}}+\sqrt{P} \sum_{i=1, i \neq k}^{K} \boldsymbol{h}_{\boldsymbol{i}} \boldsymbol{w}_{\boldsymbol{i}} \boldsymbol{s}_{\boldsymbol{i}}+n
$$

The first term of (2) is the desired signal and the second term is the interference. Zero-Forcing (ZF) beamformer and Maximum Ratio Transmission (MRT) are the linear beamforming schemes widely mentioned in literature. ZF cancels out the inter-user interference at each user while the MRT maximizes the signal gain at the intended user. The beamforming matrices of $\mathrm{ZF}$ and MRT are [8]:

$$
\begin{gathered}
Z F: W=H^{H} \cdot\left(H . H^{H}\right)^{-1} \\
\text { MRT: } W=H^{H}
\end{gathered}
$$

The SINR of the $k^{\text {th }}$ user, computed from equation (2):

$$
S I N R_{k}=\frac{P\left|\boldsymbol{h}_{\boldsymbol{k}} \boldsymbol{w}_{\boldsymbol{k}}\right|^{2}}{P \sum_{i=1, i \neq k}^{K}\left|\boldsymbol{h}_{\boldsymbol{k}} \boldsymbol{w}_{\boldsymbol{i}}\right|^{2}+N_{0}}=\frac{\rho\left|\boldsymbol{h}_{\boldsymbol{k}} \boldsymbol{w}_{\boldsymbol{k}}\right|^{2}}{\rho \sum_{i=1, i \neq k}^{K}\left|\boldsymbol{h}_{\boldsymbol{k}} \boldsymbol{w}_{\boldsymbol{i}}\right|^{2}+1}
$$

Here, $N_{o}$ is the noise power and $\rho$ is the $\operatorname{SNR}\left(P / N_{o}\right)$. Considering that the base station is using $m(1 \leq m \leq M)$ antennas to serve $k$ users, the SINR of ZF and MRT are derived from (3), in [9]. The expressions for SINR of ZF and MRT are given by (4):

$$
\begin{aligned}
& \operatorname{SINR}_{\mathrm{ZF}}=\frac{\rho(m-k+1)}{k} \\
& \text { SINR }_{\mathrm{MRT}}=\frac{\rho \cdot(m+1)}{(k-1) \cdot \rho+k}
\end{aligned}
$$


As shown in fig. 3, consider the scenario of C-RAN, where the base station functionalities are split into CU and RRU connected via fronthaul having maximum link capacity of $\varphi$. With different splits, the information transferred between CU and RRU is different and thereby, the fronthaul bandwidth requirements are different. Hence, the position of the split decides how many users' information can be simultaneously carried on the fronthaul.

\section{WIRELESS SUM RATE MAXIMIZATION}

Achievable data rate is one of the best methods to quantify the system performance, and for a single user, it is defined as the maximum rate at which transmitter can transmit over the channel. From Shannon's theorem, achievable data rate for a single user is written as: $C=\log _{2}(1+S N R)$ bits $/ \mathrm{s} / \mathrm{Hz}$

In multi user environment, interference plays a major role and hence SINR must be used instead of SNR. In such a case, the sum achievable data rate (or sum rate) is written as:

$$
C_{\text {sum }}=k \cdot \log _{2}(1+\operatorname{SINR})
$$

From equations (4.1), (4.2) and (5), we have:

$$
\begin{gathered}
C_{Z F}(k, m)=k \cdot \log _{2}\left(1+\frac{\rho(m-k+1)}{k}\right) \\
C_{M R T}(k, m)=k \cdot \log _{2}\left(1+\frac{\rho \cdot(m+1)}{(k-1) \cdot \rho+k}\right)
\end{gathered}
$$

The optimal number of users served at each functional split can be obtained by maximizing the wireless sum rate subjected to constraints of beamforming technique used and maximum fronthaul data rate. The motivation for this work is derived from [10] where a similar approach was followed for PHY/MAC split (option 6). According to theory of mMIMO, the number of antennas used must be greater that the number of users $(k \leq m)$ [4]. From the throughput calculations for each functional split, provided by [11], it can be observed that, Fronthaul bit rates of all the splits are proportional to the number of antennas. Based on these calculations and [12], required fronthaul data rates with single transmit and receive antennas are calculated and presented in Table I.

Table I: Fronthaul data rates with single transmit and receive antennas
\begin{tabular}{|c|c|c|}
\hline $\begin{array}{c}\text { Split Option } \\
\text { number } \\
\text { Fronthaul rate }\end{array}$ & $\begin{array}{c}\text { Uplink Fronthaul } \\
\text { rate }\end{array}$ \\
\hline 1 & $75.5 \mathrm{Mbps}$ & $48 \mathrm{Mbps}$ \\
\hline 2 & $75.5 \mathrm{Mbps}$ & $48 \mathrm{Mbps}$ \\
\hline 3 & $75.5 \mathrm{Mbps}$ & $48 \mathrm{Mbps}$ \\
\hline 4 & $75.5 \mathrm{Mbps}$ & $49 \mathrm{Mbps}$ \\
\hline 5 & $76 \mathrm{Mbps}$ & $49 \mathrm{Mbps}$ \\
\hline 6 & $86.5 \mathrm{Mbps}$ & $276 \mathrm{Mbps}$ \\
\hline 7.1 & $466.5 \mathrm{Mbps}$ & $451.5 \mathrm{Mbps}$ \\
\hline 7.2 & $537.5 \mathrm{Mbps}$ & $461 \mathrm{Mbps}$ \\
\hline 7.3 & $983 \mathrm{Mbps}$ & $983 \mathrm{Mbps}$ \\
\hline 8 & $1228.8 \mathrm{Mbps}$ & $1228.8 \mathrm{Mbps}$ \\
\hline
\end{tabular}

When operating with single transmit \& receive antennas, if the $i^{t h}$ split option has a fronthaul data rate of $\theta_{i}$, then the fronthaul data rate with $m$ antennas is $m$ times $\theta_{\mathrm{i}}$, because the fronthaul data rates directly scale with number of antennas. Therefore, maximum number of antennas supported at each functional split $\left(m_{\max }\right)$ is given by the integer less than $\varphi / \theta_{\mathrm{i}}$,

$$
m_{\max }=\left\lfloor\frac{\varphi}{\theta_{i}}\right\rfloor
$$

where $\varphi$ is the maximum link capacity of the fronthaul link. Thereby, the maximum number of users $\left(k_{\max }\right)$ will not be greater than $m_{\max }$. Table II shows the maximum number of antennas supported at each functional split with fronthaul link capacities of $10 \mathrm{Gbps}, 25 \mathrm{Gbps}$ and $50 \mathrm{Gbps}$, on the downlink It can be observed from Table II, that it is difficult to use large number of antennas with the spit options involving PHY, because these splits require huge bandwidth. Number of antennas that can be supported increases when we move to higher split options. The number of users served is limited by the number of antennas supported.

Table II: Maximum number of antennas supported at each functional split with different fronthaul link capacities

\begin{tabular}{|c|c|c|c|}
\hline Split & $\mathbf{1 0 G}$ & $\mathbf{2 5 G}$ & $\mathbf{5 0 G}$ \\
\hline $\mathbf{1}$ & 132 & 331 & 662 \\
\hline $\mathbf{3}$ & 132 & 331 & 662 \\
\hline $\mathbf{4}$ & 132 & 331 & 662 \\
\hline $\mathbf{5}$ & 131 & 328 & 657 \\
\hline $\mathbf{6}$ & 115 & 289 & 578 \\
\hline $\mathbf{7 . 1}$ & 21 & 53 & 107 \\
\hline $\mathbf{7 . 2}$ & 18 & 46 & 93 \\
\hline $\mathbf{7 . 3}$ & 10 & 25 & 50 \\
\hline $\mathbf{8}$ & 8 & 20 & 40 \\
\hline
\end{tabular}

For ease of calculations, assume that all users experience the same average $\operatorname{SNR}$, i.e. the value of $\operatorname{SNR}(\rho)$ is a constant. If base station uses maximum number of antennas, $\left(m=m_{\max }\right)$ then $m$ in (6) and (7) can be replaced by $m_{\max }$ from (8). This will leave the sum rate dependent only on number of users, $k$. Now, a simple plot of $C(k) v s k$, with fixed values of SNR and maximum fronthaul link capacity will provide the number of users who can be served at each functional split, to maximize the sum rate. At $0 \mathrm{~dB}$ SNR and $10 \mathrm{Gbps}$ fronthaul capacity, fig. 4(a) shows the plot of $C(k) v s k$, with ZF beamforming, and fig. 4(b) shows plot with MRT beam-forming. Fig. 5(a) and 5(b) are plotted considering $10 \mathrm{~dB}$ SNR

It is evident from fig. 4(a) that, split option 1 serves the maximum number of users (i.e. 50 users) at maximum sum rate being 70.6 bits $/ \mathrm{s} / \mathrm{Hz}$. Under the same conditions if MRT is used, split option 1 serves the maximum number of users (i.e. 132 users, equal to maximum number of antennas supported) at maximum sum rate being $77.5 \mathrm{bits} / \mathrm{s} / \mathrm{Hz}$. So, at these conditions, MRT serves the maximum users. It is 
observed that, at SNR of $0 \mathrm{~dB}$, MRT serves maximum number of users.

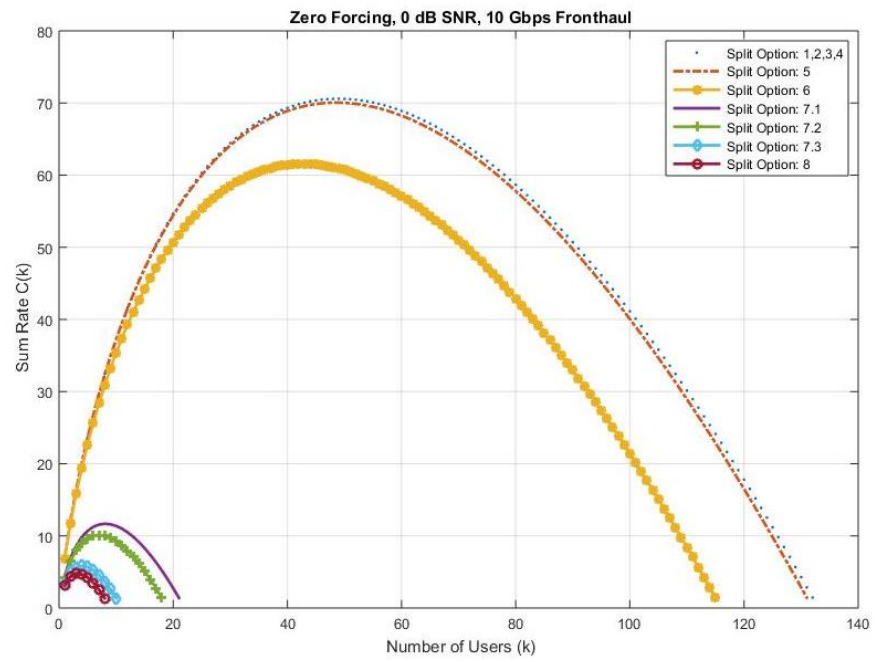

Figure 4(a): ZF and OdB SNR

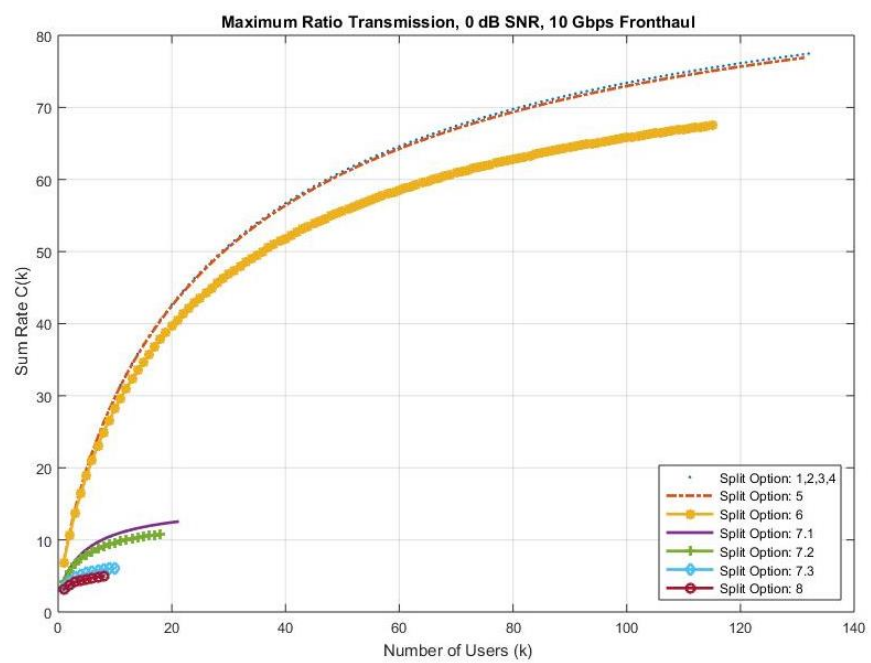

Figure 4(b): MRT and 0dB SNR

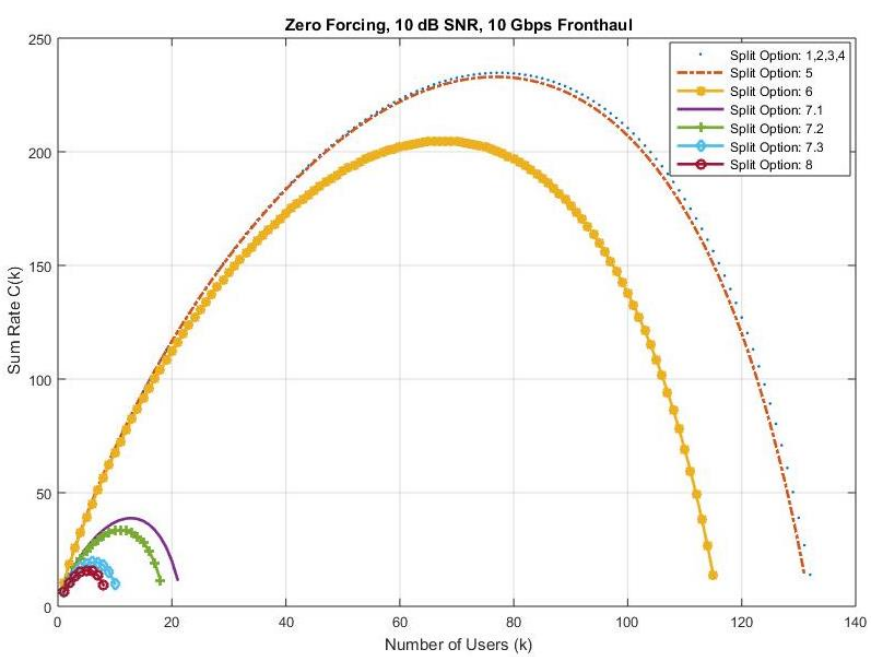

Figure 5(a): ZF and 10dB SNR

As SNR increases, ZF outperforms MRT, as shown in fig. 5. At $10 \mathrm{~dB}$ SNR, ZF beamforming with split 1 serves maximum number of users (i.e. 78 users) at maximum sum rate of 234.7 bits/s/Hz. From fig. 5(b), it can be observed that by using MRT, split 1 serves the maximum number of users
(132 users, equal to maximum number of antennas supported) at maximum sum rate being $123.8 \mathrm{bits} / \mathrm{s} / \mathrm{Hz}$.

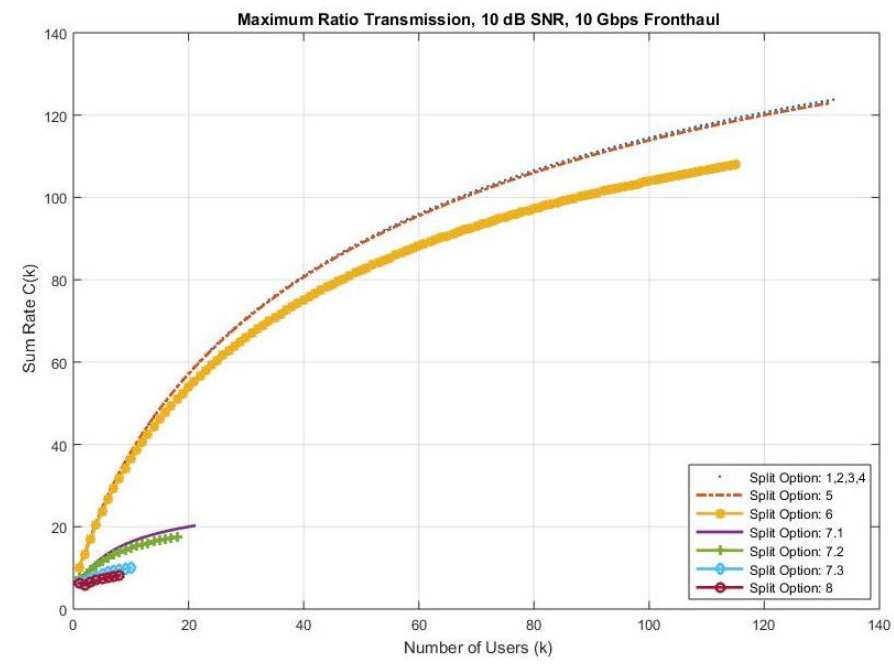

Figure 5(b): MRT and 10dB SNR

It can be observed that $\mathrm{ZF}$ provides a higher sum rate than MRT, when there is an improvement in SNR.

\section{CONCLUSION}

In this paper, the effects of implementing massive MIMO, at different functional splits of C-RAN were investigated based on maximum number of antennas supported, and optimal number of users served at each split. The huge fronthaul bandwidth requirement restrains the use of large number of antennas with the spit options involving PHY. As we move towards split option 1 , the number of antennas that can be supported increases. It is also observed that, at SNR of $0 \mathrm{~dB}$, MRT serves the maximum number of users for any split option, and at SNR of $10 \mathrm{~dB}, \mathrm{ZF}$ outperforms MRT.

\section{REFERENCES:}

[1] Y. Benchaabane, N. Boujnah, and F. Zarai, "5G Cellular: Survey on Some Challenging Techniques," in 18th International Conference on Parallel and Distributed Computing, Applications and Technologies (PDCAT), 2017. [2] A. Checko et al., "Cloud RAN for mobile networks-A technology overview," IEEE Commun. Surveys Tuts., vol. 17, pp. 405-426, Sep. 2014 [3] I, C.L., Rowell, C., Han, S., et al.: 'Toward green and soft: a 5G perspective', IEEE Commun. Mag., 2014, 52, (2), pp. 66-73

[4] Hassan, N, Fernando, X. Massive MIMO Wireless Networks: An Overview. Electronics 2017, 6, 63

[5] Kenji Miyamoto, Shigeru Kuwano et.al, "Analysis of mobile fronthaul bandwidth and wireless transmission performance in split - PHY processing architecture", Opt Express. 2016 Jan 25;

[6] Line M.P Larsen et al., "A Survey of the Functional Splits Proposed for 5G Mobile Crosshaul Networks", IEEE Commun. Surveys Tuts., Oct. 2018 [7] "Massive MIMO and Beamforming: The Signal Processing Behind the...". [Online]. Available: https://www.analog.com/en/analog-dialogue /articles/massive-mimo-and-beamforming-the-signal-processing-behindthe-5g-buzzwords.html. [Accessed: 13-Jan.-2019].

[8] Parfait T, Kuang Y, Jerry K. Performance analysis and comparison of ZF and MRT based downlink massive MIMO systems, 2014 Sixth International Conf on Ubiquitous and Future Networks (ICUFN). IEEE Press, 2014:

[9] C. Lee, C.-B. Chae, T. Kim, S. Choi, and J. Lee, "Network massive MIMO for cell-boundary users: From a precoding normalization perspective," 2012 IEEE Globecom Workshops, 2012

[10] S. Park, C.-B. Chae, S. Bahk, "Before/after precoded massive MIMO in cloud radio access networks," Proc. IEEE Int. Conf. Commun., June. 2013

[11] Small Cell Forum, "Small cell virtualization functional splits and use cases", 159.07.02, January, 2016.

[12] "R3-161813: Transport requirement for CU \& DU functional splits options." 3GPP 